\title{
Understanding The Factors Underlying Ethical Organizations: Enabling Continuous Ethical Improvement
}

Vicky Arnold, (E-mail: Arnold@coba.ttu.edu), Texas Tech University

James C. Lampe, (E-mail: odlam@coba.ttu.edu), Arthur Andersen Professor of Accounting, Texas Tech University Steve G. Sutton, (E-mail: sutton@coba.ttu.edu), Texas Tech University

\begin{abstract}
Over the past decade, issues surrounding corporate ethics and the ethical decision making of individuals in business have been a major focus in the popular press, academic research and curriculum revision efforts in business schools. One of the quandries faced throughout these efforts is how to enable an individual to make ethical decisions. In this paper, a model is proposed that focuses on organizational culture as the means by which to provide an environment that fosters ethical decision making by the organization's members. A four-stage model for understanding the current ethical state of organizations is formulated. Subsequently, the focus shifts to the underlying factors and situations that may intentionally or unintentionally exist and that may cause an organization to progress to a higher stage or regress to a lower stage.
\end{abstract}

\section{Introduction}

$y$ n recent years, academics, the press, shareholder groups, and countless other groups and organizations have released an outcry to improve business ethics. With this outcry has come an increased focus on employee training, corporate codes of ethics, and new ethics assurance services provided by the accountancy profession. In many cases, organizations have made commitments to improve the ethical decision making of their management in order to become "more ethical organizations." Unfortunately, the ethics Holy Grail that will instantly provide the finder with eternal ethical life has

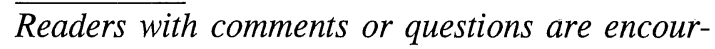
aged to contact the authors via e-mait. not yet been found. Rather, often amidst the publicity surrounding proposed organizational transformations, there lies a somewhat limited understanding of the commitment required to truly become an "ethical organization." Perhaps first and foremost, organizations must learn that ethical decision making by groups must be part of their culture.

Organizational culture consists of a complex set of common beliefs and expectations held by members of the organization. These beliefs and expectations include shared philosophies, ideologies, values, assumptions, attitudes and norms (Hellriegel, et al. 1992). This set of beliefs and expectations is often tacitly under- 
stood by members of the organization and guide their actions under a broad set of circumstances (Camere \& Vapsalainen, 1988). Culture permeates the organization and can foster an environment where ethical decision making is the norm. Conversely, culture can promote an environment in which unethical decision making is accepted behavior.

The importance of ethical decision making being dictated by organizational culture is embedded in the slow evolutionary manner in which cultures change. The complexities of dealing with deep-rooted cultures was apparent in the Bosnia-Herzegovina conflict where hatreds were suppressed for almost five decades before freedom of choice led to the freedom to once again hate. While corporate organizations have quicker turnover in membership than societies, cultural change takes years of perseveranceperhaps even decades.

The purpose of this research study is to posit and theorize stages of moral development for organizations and to develop means by which organizations can continually seek ethical improvement. This examination attempts to identify and understand the characteristics of organizations that cause some to be dominated by unethical decision making while others are dominated by ethical decision making. A four-stage model for classification of organizations is proposed and is used to generate a framework for creating ethical momentum and fostering continuous ethical improvement. The posited four stage organizational model of ethical decision making is compared and contrasted with six stages of individuals' moral development as established by Kohlberg (1976). The four stages of organizational development are separate and distinct from prior theories of individuals' moral development, but apparent parallels and differences between the two are presented to improve understanding of the posited model.

The remainder of this paper is divided into five sections. The next section overviews the differences in attitudes and activities within organizations and uses these observed behaviors to generate the four stage model. The second section compares and contrasts the four-stage classification model for ethical organizational culture with Kohlberg's theory of individual moral development. The third section focuses on why organizations may, or may not, want to implement procedures to advance to higher stages within the model. The fourth section then explores the behaviors that might be instituted by an organization wishing to encourage continuous ethical improvement in decision making and shift their organizational culture in a more positive ethical direction. The final section overviews the conclusions of the study and discusses opportunities for further applied research efforts in the area.

\section{Four Stages of Ethical Culture in Organiza- tions}

Numerous articles in both the academic and popular press have been written analyzing different organizations' ethical decision making in an attempt to document and/or understand how these organizations arrived at their current state-be it positively or negatively inclined. On the positive side, Johnson and Johnson has been perhaps the most frequently cited company and has received rave reviews for the manner in which the Tylenol scare was handled. When concerns arose as to whether someone had tampered with batches of Tylenol tablets, Johnson \& Johnson immediately recalled the potentially hazardous pain killers and shortly thereafter introduced new tamperproof packaging Greenwald 1986; Johnson 1989). The cost of recalling the pain killers was significant, raising the praise of business analysts for "doing the right thing" despite the cost (Greenwald 1986). Post hoc analyses of Johnson \& Johnson have also led to additional praise for the emphasis on ethical decision making and right behavior throughout the organization (Johnson 1989). The degree of praise certainly suggests that an organization that appears so ethical may be a very rare occurrence. 
Other companies have received scathing reviews when faced with similar situations for their unwillingness to take the action deemed appropriate by critics. For example, Ford Motor Co. has been the subject of extensive criticism over time for the manner in which they handled exploding fuel tanks in Pinto automobiles during the early to mid-1970s (Gioia 1992). Similarly, Morton Thiokal was the subject of much negative publicity after the failure of an O-ring on a space shuttle flight led to the death of all crew members and, perhaps of greatest public outcry, to the death of Christy McAuliffe, a school teacher aboard the mission for other publicity reasons. The carelessness in the O-ring manufacturing and monitoring process indicated a potential lack of emphasis on attention to safety issues throughout the organization (Elliot, et al. 1993; Sanders 1993).

More recently, Bausch \& Lomb, the subject of negative case studies and publicity, allegedly packaged the same contacts lenses in disposable packets as in the one to two year lenses for which they charged consumers perhaps 30 times the disposable price (Maremont and Barnathan, 1995). Subsequent studies have suggested that such management practices have been difficult to overcome in an aftermath effort to improve ethical decision making throughout the organization (Maremont, 1995b). Reports have suggested that the company's failure to follow through on any efforts to support ethical decision making in the past may have led to a lackadaisical culture in regards to considering the ethical implications of business decisions (Sopko, 1997).

While case studies that examine and report ethical behaviors within an organization can oftentimes be difficult to complete in an unbiased and informed manner, some trends can be found in documented actions of various companies. One recent study found that 93 percent of companies surveyed had a corporate code of conduct (Wiley 1995). The fact that so many companies have instituted such codes is not particularly earth shattering, but rather that nearly 7 percent of companies have not taken this oftentimes first step for influencing the conduct of their employees is more notable. Several other studies conducted regarding ethics implementation strategies provide additional insight into current corporate practices. Only 44.4 percent of companies surveyed conduct employee training in ethics, only 43.9 percent provide social auditing and reporting, and only 20.6 percent have undergone structural changes within the organization. In addition only 17.9 percent of companies indicate that ethics committees have been established, with appointment of an ombudsman function or establishment of a judiciary board being practically nonexistent ( 7.6 percent and 1.3 percent respectively). (Wiley 1995).

Another way in which to gain information about organizations' commitment to ethical decision making is to examine past practices and characterize activities as either passive or active. In most of the cases noted earlier, there was no overt decision by the organization to forego ethical decision making and behavior. Instead, the passive nature of the organizational approach sent such weak (or inconsistent and/or mixed) signals that ethical considerations were probably perceived as unimportant. A notable exception, of course, is Johnson \& Johnson who has instituted active procedures for encouraging positive behavior and decision making (Johnson 1989). This subtle difference between active and passive approaches can often mean the difference between organizations that are in a mode of continuous ethical improvement and those that are stagnant or experiencing declining influence of ethical considerations on organizational decision making.

In Figure 1, the bell curve is presented as a means of generalizing how organizations fall into the posited four stage model of ethical culture in organizations. This paper posits that organizations move sequentially through stages of ethical development; and, in order to change from a current stage to another stage of devel- 


\section{Four Stage Model of Ethical Culture in Organizations}

Figure 1

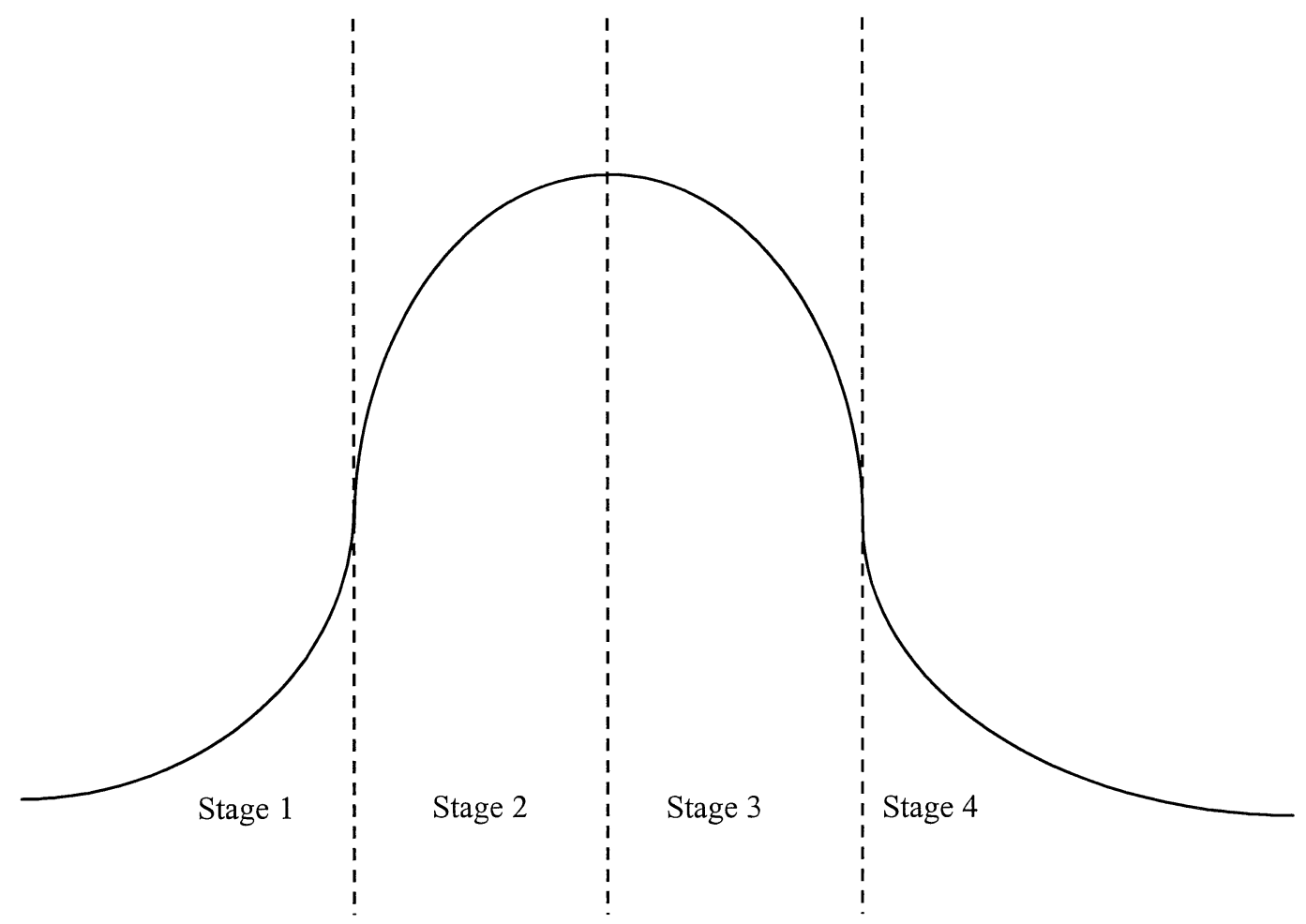


opment, an organization must transgress sequentially through the model. While used to depict the four categories, the bell curve does not necessarily provide a perfect representation of the distribution of companies falling into each category; the real distribution is not ascertainable. Probably, most companies fall into categories 2 and 3 with only a few in categories 1 and 4; and the distribution may be skewed toward 2 and 3 with the extent of skewness alternating between the two over time. Stage 1, which is the weakest form of organizational culture from an ethical decision making perspective, represents organizations that lack any intent or desire to encourage ethical decision making. Stage 2 represents a passive approach to ethical decision making where the culture within the organization desires that its employees consider ethical issues in making their decisions, but no consistent or effective procedures are in place to motivate employees to make ethically correct decisions. The culture within a Stage 3 entity promotes ethical decision making and actively pursues structures to encourage such decision making; yet in some cases, the message that the organization expects its members to make the ethically correct decision may still be inconsistent. Stage 4 organizations integrate an emphasis on ethical decision making throughout the organization; and the consistent emphasis over an extended period of time creates a climate of ethical decision making that permeates the culture. These four stages are elaborated upon in the following sub-sections.

\section{Stage 1: Absence of Intention}

As the curve in Figure 1 would suggest, the number of organizations anticipated to fall into the Stage 1 category is relatively low. Organizations primarily in Stage 1 are most frequently formed with an intent to survive by operating on the fringe of moral ground. Those companies are invariably characterized as having very low ethical standards by most individuals in society-consistent with the organizations' lack of intent or desire to encourage ethical decision making by their employees. Such organizations are often difficult to identify and are normally uncovered at the point in which they reach bankruptcy or have been exposed and are dissolving operations.

The most public recent example of Stage 1 firm behavior comes from several organizations in the tobacco industry. Revelations in congressional and senate hearings have unveiled a systematic effort by multiple firms to entrap children into smoking and to increase the addiction level of cigarettes. At the same time, these firms' top management lied about knowledge of addiction from cigarettes, denied targeting children in advertising campaigns, and denied knowledge of any link between cigarette smoking and lung cancer--all the time having extensive research results acknowledging the addictiveness and harmful effects of their products. Further, memorandums were found substantiating claims that tobacco industry firms' management were involved in an organized, intentional effort to increase the addictive power of tobacco products and to cover up information evidencing these claims. Basically, these firms sold out the public good in exchange for enhanced profitability for management and shareholders. (Shane 1997).

This is an intentionally generalized example because classifying a specific organization as falling into this category without having access to insider information that would support such a position is risky. However, the congressional and senate hearings on tobacco companies have exposed certain insider information on several industry firms providing the information for our prior discussion. Unfortunately, most experienced business persons do have personal knowledge of one or more organizations that lack the intent to operate in a fair, ethical manner. Such organizations are of lesser concern to our framework since the primary intent is to devise a method for enhancing the ethical culture of those organizations desiring to improve the ethical decision making of its members. By definition, Stage 1 organizations are not interested in 
such improvements. Hence, our focus shifts to Stage 2, 3 and 4 firms where continuous ethical improvement may be of much greater interest.

\section{Stage 2: Passive Support}

Stage 2 firms are quite different from Stage 1 firms in that a desire exists for all members of the organization to perform ethically, but there is no particular structure in place to support or encourage such behavior. These organizations are characterized by management who believe employees should be ethical decision makers, but either have never considered implementing specific policies to support ethical decision making or have failed to implement meaningful structures to encourage such behavior. For instance, the $7 \%$ of corporate entities who have not even taken the rudimentary approach of adopting a code of ethics are likely candidates to fall into Stage 2. Examples can also be noted for not-for-profit organizations such as colleges and universities. Many profit and not-for-profit entities implement a code of ethics (conduct) as an end in itself and fail to implement or support further activities. In such organizations, many employees are oftentimes not aware that a corporate code of conduct exists. Other employees may be aware that the code exists, but have no knowledge of its contents and no belief that positive/negative sanctions apply to those members of the organization that follow/break the code.

Based on available information ${ }^{1}$, Bausch and Lomb appears to be a Stage 2 company. As noted earlier, they have received extensive press and criticism over their practice of packaging the same contacts for weekly disposable use as for multi-year extended wear. This alone, would not necessarily place the firm primarily in Stage 2 versus Stage 1 or 3 . Added to this problem, however, is an apparent indifference among individuals within the organization regarding ethical issues in managerial decision making (Maremount and Barnathan 1995; Maremount 1995a; Maremount 1995b). Ethical decision making by individual members appears to be important to Bausch and Lomb management, but not a primary focus. Since the publicized debacle, efforts have been exerted to improve their image and the ethical sensitivity of the organization's individual members. In so doing, however, a deeprooted indifference to ethical decision making has been discovered (Sopko 1997). This combination suggests that the firm is primarily in Stage 2 ethical decision making and working to develop to a higher stage.

Texaco is another company that demonstrates the inconsistent messages that form within a Stage 2 company when management's actions are perceived as less than ethical. Significant public outcry followed revelations of racist comments by top management and perceived limitations on employee promotions based solely on racial discrimination (France and Smart 1996). The tarnished image that followed has impacted Texaco's business and created significant trepidation among employees (Reifenberg 1997).

At least during the 1970s, both Ford Motor Co. and General Motors would also appear to have been in primarily Stage 2 ethical decision orientation. Information surrounding the Pinto and its exploding gas tanks suggest that ethical dilemmas were treated as business cost decisions and not necessarily ethical decisions (Gioia 1992; Helms and Hutchins 1992). The same might be perceived for General Motors and the exploding fuel tanks from side impact on certain trucks (Lavin and Pearl 1994; Kerwin 1994). Both organizations generally appear to have a legitimate preference for ethical decision making. However, the histories associated with vehicle designs and subsequent reactions to public concerns indicate cultures focused (at least in some major dilemmas) on business decision making that was not particularly influenced by serious consideration of ethical issues-a typical characteristic of Stage 2 organizations.

In the same way that most business per- 
sons are aware of corporations that operate primarily in Stage 2 ethical decision making, many faculty are also aware of Colleges and Universities that act primarily as Stage 2 organizations. Examples include administrative and staff personnel who intentionally falsify hours and costs associated with extramural grant funding, known tolerance of sexual harassment, or promotion and pay decisions knowingly based on other than merit criteria.

\section{Stage 3: Active Pursuit}

Active pursuit of increasingly ethical decision making by an organization usually results from one or more of three motivations: (1) industry driving factors, (2) competitive advantage, or (3) an inherent desire to be an ethical organization. Industry driving factors provide motivation when a given industry is expected to achieve a higher moral ground or when competitors in the industry force such a higher * ground. Competitive advantage becomes a motivation when a firm perceives an opportunity to gain an edge by taking a higher moral ground. Inherent desire simply arises in some circumstances by a company's owners or management desiring to operate in an ethical manner. In all three cases, a firm may still suffer from some inconsistencies that limit its ability to achieve Stage 4 .

Industry driving factors most frequently occur in service and professional industries. One such case would be public accounting where professional standards dictate a certain level of achievement and the profession's value rests on projecting a professional and ethical aura. While there certainly may be differences in firms' positions within this stage, most of the major accounting firms should fit here. For instance, KPMG Peat Marwick has received significant publicity for its relatively new ethics assurance services (Petzinger 1996). In implementing its marketing plan for such services, firm members became indoctrinated in the importance of ethics to clients and the advantages that may exude.
However, a conscious decision was made to shift their ethics consultancy and expertise from inhouse (i.e., for firm members) activity to primarily a client service-perhaps missing an opportunity to create greater momentum toward becoming a Stage 4 organization. On the other hand, Coopers and Lybrand took the approach of developing their ethics practice as an in-house service first. All employees are required to participate in short seminars that aid in identifying ethical issues surrounding the audit decision environment (Kipp 1997). This is one such method that might be used by a firm wanting to sustain a very active pursuit of ethical decision making and perhaps reach a Stage 4 level of total integration.

Monsanto is an example of a company that actively pursues an ethical orientation for competitive purposes. In the early 1990s, Monsanto began to implement several advances in emissions control in an effort to voluntarily reduce the effect of its production processes on the environment (The Economist 1991). Monsanto was able to use this voluntary good deed in its advertising and publicity in order to create a public perception of a very ethical organization that cared about the environment and public good. The Economist (1991) noted, however, that Monsanto's actions were consistent with current efforts by several companies to take first strike action in defining new environmental goals for an industry. This enabled Monsanto to establish industry goals and to take advantage of their size and corresponding economies of scale to force new environmental standards that would be more costly for their competitors to implement. While Monsanto made apparent strides towards being an even stronger ethical organization, writings have suggested that this may not necessarily have been the intent and the momentum may not exist to move further forward. This lack of forward motion may occur because the intent was to gain business advantage rather than an inherent desire to strive for continuous improvements in ethical decision making. 
Many faculty members reading this manuscript can also recognize Colleges and Universities that demonstrate primarily Stage 3 ethical decision making. One example is when schools that require all faculty and management to participate in annual training about the organization's code of conduct, sexual harassment, and/or diversity in admissions and hiring. Another example is high ranking administrators who consistently talk and demonstrate the importance of ethical decision making with open communication lines that provide observable rewards for positive ethical behavior by faculty and staff as well as known sanctions for unethical activities.

Ben \& Jerry's Ice Cream is an apparently successful example of an organization that has transitioned from a Stage 3 to Stage 4 ethical decision making culture. They have chosen $a c$ tive pursuit of ethical decision making primarily because of an inherent desire by the founders and owners to operate an ethically and socially conscious organization. In such cases, the leadership from the top creates a momentum throughout the organization (Miller 1995; Wiesendanger 1993). The ability of Ben \& Jerry's Ice Cream to initiate this focus from the initial formation of the organization has allowed the owners to steer the organization toward a particularly strong ethical focus. When an active pursuit of an ethical organization becomes the focus of the leadership from the beginning or at least for an extended number of years, ethical decision making becomes integrated throughout the organization and may allow achievement of a rather elusive Stage 4.

\section{Stage 4: Total Integration}

While Ben \& Jerry's is demonstrative of a firm that has created an active pursuit of the ethical organization, they also appear to exemplify a Stage 4 organization. The primary difference between a Stage 3 and Stage 4 organization is the consistent application of an ethical orientation to decision making throughout the organiza- tion, at all levels of employees, and over an extensive period of time. This is not an easy level to attain and few organizations ever reach this stage as illustrated by the curve in Figure 1.

Achievement of this level requires the complete dedication of all levels of management to an unswaying, continuous focus on ethical decision making. Most organizations will fall short of Stage 4 because of periodic failures by management to "practice what it preaches." In other words, management will frequently make decisions in which the emphasis on ethics may appear to have been either ignored or even violated. If management is not careful, even if they act in an ethical manner, the perception may not be the same to observers and the inconsistencies will lead to digression by lower level management and/or employees. This digression will cause a firm to backslide into Stage 3.

These latter conditions make achievement of Stage 4 so difficult. To achieve total integration, an organization must monitor the decision making of all members of the organization and concurrently assure a reward structure consistent with ethical decision making in all aspects of operations. The consistent message to all members emphasizes the consideration of ethical issues and how that consideration has influenced organizational choice.

The Ben \& Jerry's example, while quite phenomenal, is different from the track most companies must take to achieve Stage 4 . Namely, few organizations have the foresight to choose an advocacy position for ethically and socially conscious decision making (and emphasize hiring employees that facilitate those objectives) from day one of formation. Rather, most organizations find themselves with a firmly established culture before the decision to pursue higher levels of ethical decision making across the organization is made. This necessitates a complete change of organizational culture--a more challenging and time-extensive process. 
As noted in the introduction, Johnson \& Johnson is probably the best recognized company by the popular and academic press in terms of having established total integration of ethical decision making within the organization. Highly touted for their handling of the Tylenol scare, they have leveraged this publicity with an already strong ethical culture to create what has been purported to be a model business culture for supporting ethical decision making (Johnson 1989). As predicted by the four stage model, this culture has evolved from a long-term emphasis on ethical decision making, coupled with a consistent message from management both in appearance and in action, that has lead to the total integration of a focus on ethical decision making--a model for other organizations.

\section{Organizational Vs. Individual Stages Of De- velopment}

The four-stage ethical model for organizational culture is generally consistent with parallel theories of individuals' moral development, but also has several contrasting concepts. The purpose of this section is to compare and contrast the organizational model presented here to Kohlberg's model of individual moral development. While Kohlbergian stages of moral development for individuals are not universally accepted, they have been formulated over many years and utilized by many ethicists. These individual stages of moral development provide a set of benchmarks to which the organizational stages of ethical development can be compared and contrasted in order to better describe the posited model. A central basis of the Kohlbergian formulation is that "...all individuals in all cultures go through the same order or sequence of gross stages of moral development, though varying in rate and terminal point of development" [Kohlberg 1976, pg. 176]. This cognitive developmentalist model is strongly correlated with individuals' formal education (Rest 1986, pg. 33). By contrast, the posited four stage organizational model is based on changes in the organizational culture rather than cognitive development. The four stages of ethical development within organizations posited in this paper will be compared with the six individuals' stages within the three levels formulated by Kohlberg. Aspects of the fundamental differences between an individual's cognitive developmental basis and an organization's cultural change basis for ethical decision making are to be explored further.

In Kohlberg's pre-conventional level of individual development, the focus for right vs. wrong behavior is on one's self. In Stages 1 and 2 , right behavior is determined primarily by the physical consequences of actions. Self satisfaction of needs and wants is the primary motivation for activity with only occasional consideration of others' rights or needs, and only when reciprocity is involved. This pre-conventional level of individual development can be compared with Stage 1 of organizational development characterized as absence of intention. Contrary to a cultural desire to achieve higher ethical behavior, the organization is almost purely oriented toward profit or other maximization of self benefit. Little or no consideration is given to the organization's impact on other individuals or organizations. The only reason for a Stage 1 organization to restrict unethical activity is to avoid physical or legal sanctions. As indicated by the title, the organizational culture lacks any intent or desire to seek right actions including consideration of the rights, needs, or wants of other individuals or organizations. Such rights of others are considered only when reciprocity or greater benefit to the organization is seen to accrue from it.

The key difference is that an individual progresses from pre-conventional to conventional decision making via increased levels of cognition accompanying passage of time in gaining experiences and increased levels of education. Organizations do not similarly enjoy "automatic" increases over time following additional experiences. Specific actions by management to change norms in the organizational culture must occur for increased development of organizational de- 
cision making.

Another related and key difference between Kohlberg's cognitive developmentalist model and the posited organizational model is that of retrenchment. Once an individual has achieved a higher order of cognition and a related increase in moral development, retrenchment will not occur. During the remaining active decision making time of the individual, development will either continue to progress with additional cognitive growth or the stage will plateau (Rest 1986, pg.34). Organizations, on the other hand, experience continuing changes in personnel, policies, and leadership. Changes in the organizational decision making are expected to follow perceived changes in the cultural norm -- either up or down.

In the second or conventional level of Kohlbergian individual development, the focus is on the group rather than self. In a Kohlbergian Stage 3 orientation, the decision maker defines right behavior in terms of what is pleasing to and approved by others in society, while Stage 4 behaviors are based on fulfilling one's duty and respecting both the authorities and social orders determined by the society as a whole. This is also referred to as a law and order stage of reasoning. Similarly, the posited Stage 2 of organizational ethical development in this study is characterized by passive support of ethical decision making by members of the organization. The culture in these firms is to seek a higher level of ethical activity and to act in a responsible manner pleasing to other individuals and organizations, but is premised on externalized means of achieving these goals. Taken as a whole, the organization has not developed sufficiently to have internalized right behavior. Prior self-centered activities are restricted or prevented by rules or formally developed checks and balances rather than internalized belief in right action. Incentives for, and executive management examples of, ethical behavior have not been strong enough, consistently observable, and/or in place long enough for most members through all ranks of the organization to have internalized a belief in right behavior. Guidance to higher moral activity is provided by external rules, programs, incentives, and/or monitoring.

Another parallel between stages of individual and organizational moral development is concurrent reasoning within multiple stages. An individual is not a $100 \%$ Stage 3 societal oriented decision maker that then suddenly becomes a $100 \%$ Stage 4 law and order oriented decision maker. Instead, the progression from Stage 2 to Stage 3 to Stage 4 involves reasoning from all the different levels. When the majority of the reasoning used by an individual progresses from Stage 3 to Stage 4, the individual is classified as a Stage 4 decision maker even though Stage 3 and Stage 5 reasoning is concurrently present. Organizational cultures are equally or even more accurately characterized by multiple different stages of concurrent reasoning although only one can be ascribed as the most prominent. Different divisions, different departments, different individuals, and even the same individual within an organization may display different stages of ethical decision making within a very short time span or in reaction to different stimuli. This dynamic volatility of decision bases makes it difficult to clearly classify an organization at one level of ethical development.

The third or post-conventional level of Kohlberg's model of moral development is focused on abstract, self-chosen principles. Stages 5 and 6 within this level are also referred to as the degree or percentage of principled reasoning invoked by an individual in ethical decision making. In Stage 5 of individual development, right actions are defined in terms of basic individual rights by all members of society as well as the rules, norms, and standards generated by and for society. Right behavior is guided and motivated by personal values and opinions held by each individual faced with ethical decisions. A parallel is drawn here between the Kohlbergian Stage 5 of principled reasoning with the posited organizational Stage 3 characterized by active 
pursuit of ethical decision making. A necessary component of organizational development within this stage is the inherent desire from all or nearly all of the organizational members to develop a higher moral climate. The organization as a whole must search for right behavior based on internalized values rather than on externally imposed rules, programs or sanctions.

In the Kohlbergian formulation of individuals' moral development, few people progress to a substantial level of Stage 6 reasoning. At this level, behavior is guided by abstract principles centering around justice and dignity for all members of society. These self-chosen and internalized principles far exceed and remain independent of concrete rules by the social order. In a similar fashion, very few organizations will develop to a Stage 4 level of ethical behavior in which total integration of superior ethical activity is consistently applied by all levels of management and virtually all employees for nearly all operational activities. Consistent active pursuit of this goal with pro-active organizational support at all levels is required over an extended period of time in order to achieve this total integration.

Continuous ethical improvement through the stages of organizational development is an arduous and costly long term task. Furthermore, the results most often cannot be immediately or directly observed. Even with an active desire and willingness to seek higher ethical ground, most organizations have difficulty in knowing how to achieve and how to measure continuous ethical improvement.

\section{Choosing The Route To Continuous Im- provement}

Making a commitment to incorporate continuous ethical improvement processes is not an easy choice. Many organizations (if not most) do not have extensive improvement processes in place for a variety of reasons. The most prevalent reasons fall into three categories: (1) a lack of concern for and/or awareness of how to pro- mote ethical decision making, (2) insufficient desire or ability to invest the resources required to design, install, and maintain a program of continuous ethical improvement, and (3) management's desire to retain flexibility in decision making without the constraints of having to consistently act in an ethically oriented manner.

One of the primary objectives of this research is to directly deal with the first issue-raising the understanding of the importance of ethical decision making to long-term organizational success and increasing the awareness of methods organizations can use to promote ethical decision making via cultural change. Categories two and three are much more difficult to counteract, and the focus at this point will be to understand why some organizations make these choices.

Clearly, implementation of a continuous ethical improvement program requires a major investment by the organization. One approach being taken by several organizations is to hire a consultant or an ethics auditor (Abdolmohammadi 1997). KPMG Peat Marwick has been the subject of broad coverage for their development of an ethics assurance services practice designed to help companies assess and improve the ethical focus of their organizational decision making (Petzinger 1996). Other organizations have adopted approaches more similar to that of Coopers and Lybrand. In this scenario, one or more individuals are employed full-time to provide training to employees on identifying and understanding ethical dilemmas within there own operations as well as those of clients (Kipp 1997). The costs are quite high, both in terms of the salaries and support of the trainers, and the "non-productive" time required for staff to participate in training sessions. Hence, the total cost of implementing a meaningful continuous ethical improvement process will be substantial for most organizations.

The third limitation (e.g., not having complete decision making flexibility) should not 
be particularly surprising given that U.S. corporate management has long been criticized in the management literature for not being sufficiently focused on long-term viability. Rather, the focus tends to be on short-term goals that will keep stockholders happy. This short-term focus is incongruent with the long-term consistent emphasis on ethical decision making necessary to implement an effective continuous ethical improvement process. This incongruence is likely to be a major barrier for many organizations.

In light of these arguments, perhaps the next logical question is, "Why would an organization pursue a strong continuous ethical improvement system?" Three reasons were noted earlier why some firms may wish to be ethics leaders: (1) industry factors, (2) competitive advantage, and (3) an inherent principled desire for an ethical organization. However, these reasons are more likely to be applicable to organizations that are already in the upper half of the bell curve. The question still remains, "What is the advantage to lower stage firms?"

Clearly, Stage 1 firms are unlikely candidates; however, many (if not most) Stage 2 firms never intended to find themselves on the lower ends of the ethical decision making spectrum. As such, Stage 2 organizations oftentimes get an unexpected wakeup call when unethical decisions lead to negative consequences--be it identified fraudulent activities or things such as known design flaws in automobiles that lead to exploding gas tanks or other safety hazards. These organizations suddenly find themselves in the very challenging position of reversing an out of control, downward spiral on the organizational ethics front.

Similarly, one of KPMG Peat Marwick's big selling points for their ethics assurance services practice has been as a defense mechanism against criminal and civil charges for the management of risky organizations. The perception is that, if a company can demonstrate efforts to assure against unethical decision making, federal sentencing guidelines may allow for a lighter sentence based on demonstrated efforts to alleviate the illicit behavior assuming such an event should later occur or be discovered (Petzinger 1996).

Finally, there is a marketplace for socially responsible firms; and there is undoubtedly some advantages to attracting this market. Similarly, product sales can be expected to benefit in many industries for those companies that can create an image of being ethically and socially conscious such as Saturn automobile who promotes their reputation in media advertisements. For most companies there is some benefit to achieving this image; the question is whether it is worth the cost.

\section{Cultural Change Through Continuous Ethical Improvement}

If an organization makes the decision to pursue a more ethical orientation to its decision making processes, the next step is to determine the structures that must be implemented to facilitate such improvement. As with any continuous quality improvement effort, the organization must complete a self-assessment of its current level of achievement including the strengths and weaknesses of existing processes. The four-stage organizational model of ethical culture is intended to provide a benchmarking framework for organizations to identify their relative standing. Once the self-assessment has been completed, the challenge becomes how to implement appropriate structures for the encouragement of ethical improvement. Continuous ethical improvement becomes a repetitive cycle of self-assessment, development of an improvement plan, and implementation of the improvement plan as illustrated in Figure 2.

In the following sub-sections, continuous ethical improvement goals are explored for each of the four stages. Much of the theory underlying the approaches discussed in this section evolves from Arnold et al.'s [1997] work on an 


\section{Continuous Ethical Improvement Model Figure 2}

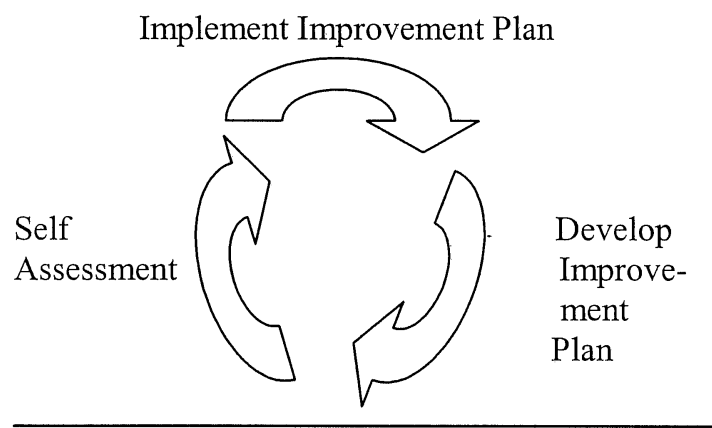

Epidemic Theory of Organizational Ethics. Epidemic Theory suggests that immoral behavior will cause exponential degrees of negative behavior and will cause firms to transcend downward through the stages (most frequently from Stage 3 to Stage 2 and/or Stage 2 to Stage 1). Conversely, positive epidemics of moral behavior will move organizations upward through the model stages with epidemics of positive behavior being a necessary (but not sufficient) precondition for achieving Stage 4. Positive epidemics are most useful in transcending from Stage 2 to Stage 3 and Stage 3 to Stage $4 .^{2}$

\section{Transitioning Between Stage 1 and Stage 2}

Stage 1 is the most difficult stage of the framework for an organization to transcend. At Stage 1 , most organizational members will have been exposed to a negative virus of demonstrated unethical behaviors that are accepted by the firm. Many organizational members will regularly push the thresholds of illicit or unethical behavior. Those who have not participated in such behavior will have learned to either condone or look the other way when such behaviors occur or will choose to leave the organization. Hence, the idea of an epidemic of unethical behavior that spreads much the same way as a disease epidemic.

Attempting to cure an epidemic of un- ethical decision making in a Stage 1 firm is not much different than dealing with a disease epidemic that is out of control. First, drastic measures must be taken to slow the spread of the undesired disease; then further measures to start promoting health must be implemented. In many cases, quarantining infected individuals may be the only solution. In other words, as infected individuals are identified (even if in the majority), they must be removed from contact with the uninfected members of the firm-typically through either firing the individuals or providing sensitivity training before allowing them to reenter the work environment. Such ethical sensitivity training will be an attempt to cure the individual of the disease; and, the individual must remain under close examination to identify any relapse into unethical behavior. Relapse may result in further treatment (i.e., training) or permanent quarantine (i.e., job termination).

Improving the ethical culture of an organization by introducing an ethic epidemic represents a slow process. The first thing that must occur is slowing the rate of increase of the ethics virus. Second, reducing the number of newly infected individuals should occur. Finally, the organization can begin to gain control of the unethical activity and begin to transcend the stages of development.

In order for a treatment program to work effectively, structures must be put into place to identify infected individuals and, in particular, to identify initial contacts with the virus of unethical behavior. If organizational members ingrained to unethical behavior are not removed, intensive and significant procedures will be necessary to reorient the perception of cultural norms. This may mean a complete stand down of normal production or operations in order to emphasize the importance of ethical training and to introduce a change in culture to a desire for more ethical activities. A variety of structures can help in this process. Examples of useful structures for initiating disease control for unethical behavior follow. First, introducing a 
meaningful code of conduct that is clearly communicated to members of the firm. Second, providing training either through required reading materials or preferably through basic training on the code of conduct and minimum acceptable levels of behavior. Third, organizing meetings between members of management who have received training and work groups associated with the managers in order to spread the message. Fourth, identifying failures in the system where unethical behaviors have persisted, coupled with subsequently using these example failures to explain why the given behaviors are not acceptable. Fifth, using anonymous hot lines to provide the organization's members with a means to report ethical violations without the risk of repercussions. Sixth, applying positive/negative sanctions to promote/punish various behaviors. Implementing effective control is most successful when combinations of multiple structures are adopted.

In short, to move out of Stage 1, an organization must recognize that unethical behavior exists in epidemic proportions and emergency measures must be taken to gain control of the epidemic and to reduce the unethical behavior of its members. Only by recognizing the epidemic and taking significant observable actions can an organization have any hope of escaping Stage 1 before disaster strikes and the organization faces serious repercussions and even possible ceasing of operations. Implementing and continually monitoring activities such as those previously listed is essential.

Likewise, the same issues can be raised in determining how an organization avoids slipping into Stage 1 from Stage 2. Stage 2 firms are particularly susceptible to epidemics because of their inattention to providing support and motivation for ethical behavior. If a Stage 2 firm does not detect a virus early as it begins to spread, the organization will be at high risk of an epidemic. Once the epidemic takes hold, preventing a slide into Stage 1 is extremely difficult and reversing the momentum becomes even more difficult-similar to the difficulties of moving any Stage 1 organization to Stage 2.

\section{Transitioning Between Stage 2 and Stage 3}

Transitioning from Stage 2 to Stage 3 (or for that matter Stage 3 back to Stage 2) is perhaps the easiest and least tumultuous transition for an organization--depending on where a firm is within the given stage. A Stage 2 firm that is at the lower end of the stage and potentially facing a decline to Stage 1 is certainly different from one that is at the upper end of Stage 2 and pursuing a move to Stage 3. As noted in the previous sub-section, a firm at the lower end of Stage 2 (as well as most firms in Stage 2) is vulnerable to a potential epidemic of unethical behavior. Given that a firm desires to increase the influence of ethics on its decision making processes, how does one move from Stage 2 to Stage 3?

The difference between Stage 2 and Stage 3 is not necessarily a desire for ethical decision making since organizations in both states desire ethical decision making. Rather, the difference is having a preference versus actively supporting and promoting such decision making. To move from Stage 2 to Stage 3, an organization must assess its current strengths and weaknesses (i.e., complete a self-assessment) in its decision making processes and determine how to actively pursue an increased organizational awareness and consideration of ethical issues during decision making. In other words, the organization must be infected with a "positive virus of ethical behavior".

The first things that Stage 2 firms must address are the inconsistent messages that employees have received over an extended period of time. In a passive ethical environment, employees often witness managers who manipulate funds to support employee parties, provide job site special meals, fund group activities, pad reimbursements, pad budgets to fund unnecessary acquisitions, cover for slacking workers, 
etc. These activities may not necessarily be wrong in many circumstances, but workers may observe management manipulating budgets and funding allocations to divert funds to activities that would otherwise not warrant support from the organization. A host of other similar activities may exist to provide conflicting messages (signals) about the importance of adherence to ethical principles.

Each such "fudging of the numbers" sends an unclear message to other employees as to the organization's commitment to ethical decision making and what constitutes ethical decision making. This confusion is certainly escalated when there are limited policies or procedures in place to aid employees in determining what actions are deemed appropriate by the organization. This confusion is extended even further when competing motivations to enhance productivity and profitability appear to dominate organizational priorities to the perceived detriment of ethical decision making. When the individuals who most blatantly fudge the ethical boundaries are deemed most meritorious of advancement or raises, the mixed message is exceptionally clear to most members of the organization. Hence, the organizational self-assessment must not only encourage ethical behavior, but also address the competing motivations that are inevitably in place as barriers to the pursuit of ethical behavior as a priority. A balance must then be struck and clearly communicated to employees regarding how ethical decision making fits into the organizational culture and will be assessed and rewarded.

A variety of structures can be put into place in order to encourage active pursuit of ethical decision making. Examples of these structures would include the following. First, actively promoting a code of conduct that includes meaningful actions by the organization such as an ombudsman function, a judiciary board or an ethics committee. Second, carefully considering the perception of whistleblowers in the organization and minimization of any per- ceived potential retribution to such behavior. Third, using anonymous hot lines to facilitate whistleblowing in circumstances where individuals may still have fears of retribution. Fourth, providing extensive training (especially middle and upper level management) either through required reading materials or preferably through basic training on the fundamentals of identifying ethical dilemmas and logically resolving such dilemmas in an ethically sound manner. Fifth, using meetings between members of management who have received training and work groups associated with the managers to spread the message. Sixth, clearly communicating the importance of ethical decision making from all levels of management throughout the organization.

Obviously, many of these same techniques were noted as having potential for promoting change from Stage 1 to Stage 2. However, each of the techniques evolves in a slightly more sophisticated manner to move the organization to new levels. Nonetheless, the similarities are symbolic of the need to remain relatively constant in promoting and supporting awareness and to consider ethical issues during decision making processes. In terms of epidemic theory, constant awareness is consistent with the concept introducing positive viruses--in this case a virus of ethical behavior--and spreading this virus throughout the organization to counteract the negative viruses that may threaten the ethical foundations of the organization.

If an organization in Stage 3 becomes overly satisfied with its position as an ethical entity, a lackadaisical perspective may develop that will ultimately diminish the active pursuit of ethical decision making. As a result, a firm in Stage 3 can easily slip back to Stage 2 by being too comfortable with the structures in place and failing to complete the continuous ethical improvement cycle on an on-going basis. The failure to reassess the strength of current activities and the failure to implement on-going support systems for ethical decision making can dilute the efforts of the organization, diminish the im- 
portance placed on ethical decision making, and ultimately lead to a decline from Stage 3 to Stage 2. Thus, the emphasis on continuous improvement should not be lost.

\section{Transitioning Between Stage 3 and Stage 4}

Transitioning from Stage 3 to Stage 4 is a commitment that often extends beyond the resources an organization is willing and/or has available to expend on efforts for additional ethical improvement. Arnold et al.'s [1997] Epidemic Theory would suggest that an all out effort to combat the last vestiges of a disease must be waged with the widespread use of vaccines and positive viruses. For organizations wishing to pursue total integration of ethics into the organizational culture, an extensive combination of programs to eradicate even occasional unethical behaviors (vaccines) and programs to promote ethical behavior (positive viruses) must be used.

Eradication of unethical behavior and infiltration of a strong commitment to the pursuit of ethical behavior is not a simple nor quick process. Eradication requires a fundamental change in the organizational culture to one which has zero tolerance for less than ethical behavior and sanctions such behavior with clear and severe consequences. Inflicting such cultural change will take years and likely decades of total commitment by management. The costs may also include a loss of organization members that have been valuable contributors to the organization except for selected unethical behaviors. Encouraging and rewarding whistleblowing activities that help weed out unethical members can be both painful and costly.

The use of positive viruses can also take an extended period of time to infiltrate an organization's culture. Typical methods would include the following. First, providing extensive training on the analysis and resolution of ethical dilemmas in business environments. Second, incorporating ethics counselors to help management in assuring a consistent message of ethical performance. Third, frequently communicating information from top management to all members of the organization regarding the importance of ethics to good business decision making. Fourth, forming ethics committees and/or judiciary boards to review organizational activities and to provide proactive recommendations on future activities and decision making processes. The key is to literally bombard the organization's members with a message that emphasizes ethical decision making as a priority. Note, however, that the type of structures that are most useful in transitioning from Stage 3 to Stage 4 are quite different from the other stages. Notably absent from the current list is also the use of hotlines which should no longer be necessary - particularly given the lack of need for anonymity in an organization fully committed to ethical decision making.

Most important to the evolutionary process at this point is that the ethics message needs to be clearly communicated on a consistent basis throughout the organization. The difficulty for a Stage 4 firm that wishes to avoid regressing to Stage 3 is how to keep the momentum. However, continuous hiring of employees that buy into an organizational culture premised on ethical decision making will help. Second, all new employees should receive initial training in ethics much the same as they would other job skills. Third, all employees should continue to have meetings on an on-going basis focused on relaxed conversations regarding how to continually improve the ethical behavior of the organization. Further, management needs to assure that its message is consistent and unwavering regarding the need for an emphasis on ethical issues throughout the decision making process.

For an organization to ever be completely in Stage 4 and/or solidly remain in Stage 4 for an extended period of time will be rare. The momentum, enthusiasm, and general culture that promotes active consideration of ethical issues in all decision making is very difficult to maintain. Management, as well as all other or- 
ganizational members, must remain focused on ethical behavior and continuously reconsider the means by which such behavior is promoted. Without such a focus, maintenance of a total integration of ethical decision making throughout the organization will be highly unlikely.

\section{Concluding Remarks}

In this paper, a four stage organizational framework for ethical culture has been presented. This framework provides organizations with the means by which to self-assess their current state of ethical decision making and commitment and provides proactive recommendations for advancing to higher level stages and defending against slips to lower stages. The framework also provides researchers with a vehicle for better understanding the nature of organizations' ethical commitment and the differences in the cultures of various organizations. Specifically, the framework attempts to examine why some organizations are successful in pursuing total integration of ethics into the organizational decision making process, while other organizations fail to demonstrate any intent to consider ethics an important part of the decision making process.

Any effort to empirically gather data for purposes of classifying individual organizations is beyond the scope of this paper. Rather, future research efforts could greatly enhance the power of the framework by providing additional evidence on various firms that fall into the different stages. This empirical work could be very beneficial in improving the strength of the benchmarking capabilities of the framework.

Other future research should go beyond simple classification of the current state of various organizations to focus on the transition between stages of the framework. This will require longitudinal study of several companies to understand how different organizations shift between stages and to determine if the predominance of organizations shift to certain stages with shifts in society as a whole. This research would be even further strengthened by pursuing in-depth case studies of individual organizations to identify the structures implemented at different points in time by the firms and the impact these structures have on the ethical evolution of the organization. For instance, at each stage several structures have been recommended to aid an organization in making a specific move towards the next stage. Little is known, however, which set of structures are most effective at certain points in time or when they are moderated by broader organizational culture variables.

Another area of potential future research would be to examine whether organizations at different stages of the model have correlated differences in financial performance. Do Stage 4 firms tend to be more profitable? Are share prices increased or decreased by upward movement through the framework? Only anecdotal data is available as to the value of ethical decision making to the long-term viability and financial position of organizations, and empirical study in this area could greatly benefit from use of the four stage framework.

Finally, interest has begun to rapidly grow in the potential for client services in the ethics area. Known efforts to develop ethics practices by KPMG Peat Marwick, Price Waterhouse Coopers, and Arthur Andersen are symbolic of the perceived opportunity. Additionally, internal audit organizations are increasingly involved in internal assurance and consultancy services in a broad range of areas where ethics seems to integrate well. For these internal and external audit organizations, an integration of this paper's four stage framework with the COSO framework for internal control would appear to have great benefit. Many of the core concepts of the two frameworks are very similar, and future research on their integration would appear to be quite fruitful.

While providing fertile ground for future research, the organizational ethics frame- 
work also provides great opportunities for practice organizations to initiate continuous ethical improvement systems. This framework provides companies with a necessary tool for completing organizational ethics self-assessments as step 1 in the improvement process. Once a self-assessment has been completed, organizations can use all or part of the recommended set of structures that have been put forth for enabling improvement from one stage to the next. Replication of the continuous ethical improvement cycle should enable companies to reach the desired stage of ethical decision making if the required organizational commitment exists.

\section{Endnotes}

1. Categorization of organizations is difficult when working with imperfect information. Nonetheless, publicly available information does provide some basis for making assumptions that certain companies may fit in this or other categories.

2. The language used with Epidemic Theory is fairly consistent with the medical science popular terminology. As such, this paper adheres to this language in the following sections with discussion of viruses, vaccines, quarantines, and so forth.

\section{References}

1. Abdolmohammadi, M.J., "The Corporate Ethics Audit: A Detailed Task Model," Working paper, Bentley College, 1997.

2. Arnold, Vicky, James C. Lampe, and Steve G. Sutton, "Creating a Morally Driven Organization: A Model for Fostering an Epidemic of Moral Intensity," Ethics and Economic Behavior in Accounting and Taxation Conference, April, 1997, Norman, Oklahoma.

3. Elliot, N., E. Katz, and R. Lynch, "The Challenger Tragedy: A Case Study in Organizational Communication and Professional Ethics," Business \& Professional Ethics Journal, Vol. 12, pp. 91-108, 1993.
4. "Environmental Protection: Goody TwoShoes," The Economist, Vol. 321, p. 68, 1991.

5. France, M., and T. Smart, "The Ugly Talk on the Texaco Tape," Business Week, November 18, 1996, p. 58.

6. Gioia, D.A., "Pinto Fires and Personal Ethics: A Script Analysis of Missed Opportunities," Journal of Business Ethics, Vol. ii, pp. 379-89, 1992.

7. Greenwood, J., "Tylenol Decisions to Cost Johnson and Johnson about $\$ 150$ Million," Business Insurance, Vol. 20, p. 2, 1986.

8. Helms, M.M., and B.A. Hutchins, "Poor Quality Products: Is Their Production Unethical?" Management Decision, Vol. 30, No. 5, pp. 35-46, 1992.

9. Johnson, C.H., "A Matter of Trust," Management Accountant, Vol. 71, pp. 1213, 1989.

10. Kerwin, K., "GM Pickups: The Issue is Safety, Not Regulation," Business Week, December 12, 1994, p. 95.

11. Kipp, S. "Ethics Training at Coopers \& Lybrand," Ethics and Assurance Services in Practice, Northeast Behavioral Accounting Research Series (NEBARS), Boston, April 1997.

12. Kohlberg, L., "Moral Stages and Moralization. The Cognitive-Developmental Approach to Socialization". In Moral Development and Behavior, ed. T. Likona. New York: Holt, Reinhart, and Winston, 1976.

13. Lavin, D., and D. Pearl, "GM Trucks Had Fuel-Tank Flaw, Regulators Find," Wall Street Journal, October 18, 1994, p. A3.

14. Maremont, M., "Bausch \& Lomb's Board Puts on its Glasses," Business Week, November 6, 1995a, p. 41.

15. Maremont, M., "Judgment Day at Bausch \& Lomb," Business Week, December 25, 1995b, p. 39.

16. Maremont, M. and J. Barnathan, "Blind Ambition: How the Pursuit of Results Got 
Out of Hand at Bausch \& Lomb," Business Week, October 23, 1995, pp. 78-82.

17. Miller, A., "Sundae School (Ben \& Jerry's Social Responsibility Claim)," Inc., December 30, 1995, p. 29-30.

18. Petzinger, Jr., T., "This Auditing Team Wants You to Create a Moral Organization," Wall Street Journal, January 19, 1996, p. B1.

19. Reifenberg, A., "Texaco Agrees to Report to EEOC on Promotion of Racial Minorities," Wall Street Journal, January 6, 1997, p. B5.

20. Rest, J.R., Moral Development Advances in Research and Theory. New York: Praeger, 1986.

21. Sanders, J.T., "Assessing Responsibility: Fixing Blame versus Fixing Problems," Business \& Professional Ethics Journal, Vol. 12, pp. 73-86, 1993.

22. Shane, S., "Criminal Probes Shadow Tobacco," The Baltimore Sun, June 20, p. 1A, 1997.

23. Sopko, J.L., "A New Focus at Bausch \& Lomb," Democrat and Chronicle, Rochester, New York, January 5, 1997.

24. Wiesendanger, B., "Annual Report Portfolio: Ben \& Jerry Scoop Up Credibility," Public Relations Journal, Vol. 49, p. 20, 1993.

25. Wiley, C., “The ABC's of Business Ethics: Definitions, Philosophies and Implementation," Industrial Management, Vol. 37, pp. 22-27, 1995. 
Notes 